\title{
Biomimetic Design of a Multi-Layered Dust Protection System for Optical Instruments Operating in the Lunar Environment
}

\author{
Matthew Davidson ${ }^{1 *}$, David Bligh ${ }^{1}$, Neil Maloney ${ }^{1}$, Carmen McKnight ${ }^{1}$, Warren Young ${ }^{1}$, \\ L.H. Shu ${ }^{2}$, Marie-Josée Potvin ${ }^{3}$, Andrew Warkentin ${ }^{1}$ \\ ${ }^{1}$ Department of Mechanical Engineering, Dalhousie University, Halifax \\ ${ }^{2}$ Department of Mechanical and Industrial Engineering, University of Toronto, Toronto \\ ${ }^{3}$ Spacecraft Engineering Directorate, Canadian Space Agency, Saint-Hubert, Québec \\ *mdavids1@connect.carleton.ca,david.bligh@dal.ca,neil.maloney@dal.ca, \\ carmen.mcknight@dal.ca,warren.young@dal.ca, shu@mie.utoronto.ca, \\ marie-josee.potvin@asc-csa.gc.ca, andrew.warkentin@dal.ca
}

\begin{abstract}
A method of identifying biologically meaningful keywords not obviously related to engineering keywords was developed to enhance discovery of relevant biological analogies for design problems. This paper reports the use of biologically meaningful keywords to identify biological analogies to generate solutions for protection required during lunar exploration. In lunar exploration, dust poses a significant problem due to its pervasiveness, adherence, and abrasiveness, causing premature failure of space suits and mechanisms. In this paper, biomimetic concepts are developed to protect a laser/telescope system. The resulting design is comprised of two subsystems. An antagonistic bending Shape Memory Alloy (SMA) actuator system, inspired by bivalves (a class of molluscs that include scallops, clams, oysters and mussels), is used to control the opening and closing of a two-piece lid system, while a high-voltage DC field generator prevents charged dust particles from approaching the optical surfaces. Preliminary results indicate that the SMA actuation system is capable of greater than one-hundred repeatable lid-opening and closing cycles. In addition, the high-voltage DC field was capable of controlling and deflecting $98 \%$ of incoming charged polystyrene particles $\left(D_{\text {mean }}=1 \mathrm{~mm}\right)$ away from a representative surface. The method of using biologically meaningful keywords to identify analogies was successfully applied in this case and could be applied in a variety of settings to generate useful solutions.
\end{abstract}

\section{Introduction}

The benefits of emulating biological phenomena in engineering design are demonstrated by many successful biologically inspired products. Approaching engineering design problems from a biological perspective often results in a more thorough investigation of solutions, including those often neglected by more traditional design approaches.

In this paper, we first summarize relevant work on biomimetic design. Next, we describe the design problem of protecting a LIDAR (LIght Detection And Ranging) system against lunar regolith (dust), which poses a significant problem due to its pervasiveness, adherence, and abrasiveness, causing premature failure of space suits and mechanisms. The use of biologically meaningful keywords to identify relevant analogies is then demonstrated for the lunar regolith problem. Finally, a prototype developed based on selected analogies is described and tested.

\section{Related Work}

Previous work on biomimetic design involved searching natural-language text for instances of engineering keywords that describe the desired function of design solutions. However, searching for instances of engineering keywords in biology texts is not always productive, as the language used between the domains of biology and engineering are sufficiently varied. Consequently, a method of identifying biologically meaningful keywords not obviously related to engineering keywords was developed to enhance discovery of relevant biological analogies for design problems [1][2]. 
Chiu and Shu demonstrate this method by identifying biologically meaningful keywords for the engineering keywords 'remove' and 'encapsulate' [1]. Using this method, Cheong et al. [2] translate terms of the functional basis (a systematic grouping of verbobject pairs meant to span the engineering domain [3]) into biologically meaningful keywords.

\section{Problem Description}

Solutions are sought for protection against regolith for a LIDAR device, similar to one used for the Phoenix Mission on Mars, shown in Figure 1. A LIDAR is an optical instrument that can detect particle concentrations kilometres above the instrument itself and consists of two major components: a highpowered laser that points upward (small light grey cylinder) and an optical receiver (large gold cylinder). The closed cover protects both the laser beam canister and the receiver lens. Improved protection is required for the system both during operation and while idle.

The challenge of protection from regolith is common to most scientific instruments used on the Moon. Solutions must accommodate both the lunar environment and lunar regolith, which prevent implementation of most obvious solutions. Therefore, creative design techniques are required to expand the range of possible solutions and identify feasible ideas.

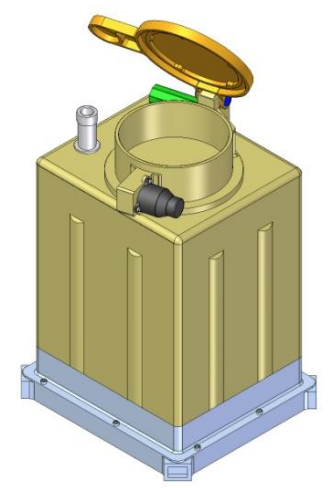

Figure 1. LIDAR system (approx. $20 \mathrm{~cm}$ in height).

\subsection{Lunar Environment}

Lunar days and nights last 14 earth days each and temperatures vary from extremes of $120^{\circ} \mathrm{C}$ during the day to $-150^{\circ} \mathrm{C}$ at night in the most common exploration areas [4]. Solutions must accommodate large temperature swings that occur rapidly during the change from day to night and vice versa. This limits the selection and arrangement of materials.

The lunar atmosphere is so thin that the environment can be considered as a hard vacuum [4]. This also affects the material selected since some materials, such as polymers, will outgas in a vacuum, causing severe physical property degradation.

\subsection{Lunar Regolith}

Lunar regolith adheres strongly to all surfaces. The mechanical aspect of adhesion is due to the jagged shape of regolith particles as shown in Figure 2. The absence of an atmosphere means there is no wind that would otherwise round regolith particles, resulting in sharp edges [5]. While Figure 2 shows a fairly large particle, most particles are below $70 \mu \mathrm{m}$ in diameter; particles in this size range can infiltrate and abrade almost all mechanical systems, and will even abrade mechanical seals [4].

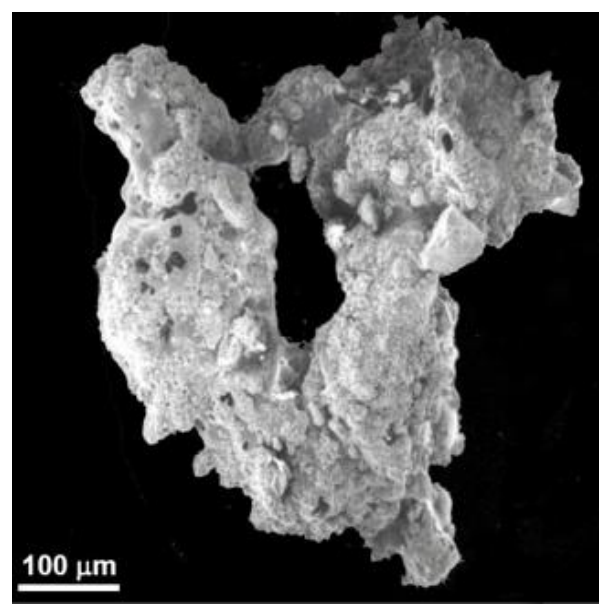

Figure 2. Lunar regolith particle.

Image Credit: David S. McKay, NASA/JSC, Permission Granted.

Electrostatic adhesion is due to positive charging of particles by solar wind during the lunar day and negative charging by plasma electron currents at night [5]. This electrostatic charging enables particles to cling to ungrounded conductive surfaces and nonconductive surfaces. Finer regolith grains will levitate under electrostatic charging and therefore, dust is present at instrument level, even in the absence of any mechanical disturbance of regolith.

\section{Concept Generation}

The engineering keyword 'protect' follows directly from the current problem statement. In the translation from engineering to biologically meaningful keywords, 'protect' is grouped with the functional basis words: 'prevent', inhibit', and 'shield'. Corresponding biologically meaningful keywords are: 
'cover', 'surround', 'inhibit', 'destroy', 'change shape', 'bind', 'release', 'attach', 'protect', 'repel', 'push away', 'shield', and 'defend'. Keywords that led to matches for this application are italicized.

More promising keywords from the preceding list of biologically meaningful keywords were selected and used to search for instances of these words in the text, Life, the Science of Biology, by Purves et al. 2001 [6]. We describe below a few of the interesting matches and how they were found and applied.

The keyword 'protect' returned several matches in the aforementioned biological text. One match was not used strictly analogously, but it did give an idea for a simple configuration of the lens cap that would result in less regolith falling onto the lens during opening. The matched phenomenon involved a "clam", and suggested a lens cap in the form of two halves of a bivalve that opens from the top, such that regolith that has collected on top is less likely to fall onto the lens than from a single hinged cap, the current configuration, shown in Figure 1.

The keyword 'repel' led to a match involving the use of charge to repel and attract. This inspired the idea of collecting and managing particles using highvoltage DC (direct current) electromagnetic fields.

\section{Selected Biological Analogies}

Promising biological analogies were selected for further investigation and development. More targeted research to reveal specific details of some of the relevant biological phenomena led to proof-of-concept prototype construction and preliminary testing.

\subsection{Bivalve Biology}

The bivalve class of mollusks, including scallops, clams, oysters, and mussels, have evolved for life in particulate-laden environments on earth. Many bivalves spend large portions of their lives submerged in sand or mud, and 'protect' themselves from both predation and environmental hazards, such as excessive particle intrusion, with their shells [7].

The geometry of deep-tunneling bivalve shells facilitates easy travel through sand and mud, while offering protection from hazards. The acute angle (10$20^{\circ}$ ) where the two valves (shell halves) meet deflects sand away from mating surfaces, and the curvature of the shell itself sheds passing sand particles.

It is also important to note that the two valves pivot to open and close the shell using a flexible ligament [7]. This ligament functions as a hinge and is resistant to particle fouling by eliminating rotational sliding motion between the valves.

\subsection{Bivalve Inspired Solutions}

For the bivalve, both the curvature and attachment of the shell play important roles in its defense. Therefore, both the bivalve-inspired curvature and joint were incorporated into a two-piece cover system. The curvature of the lids is important in shedding bulk amounts of regolith and preventing regolith from falling onto the lens area upon opening.

The joint found in bivalves inspired the development of a Shape Memory Alloy (SMA) actuation system, which mimics the ligament system found in these organisms. The SMA hinge was developed in an effort to eliminate the relative motion found in traditional rotary joints, thus increasing resistance to particle fouling.

\subsection{Relevant Physiology of the Human Eye}

Although not a product of the aforementioned keyword search, an obviously analogous biological phenomenon for the protection of a visual sensing system from dust is that of the human eye. In the eye, different types of tears, secreted by the lacrimal and meibomian glands, are used to hydrate the eye and provide a barrier between dust and the sensitive tissues of the eye [8].

This mechanism is used in conjunction with the eyelid, which provides a sweeping action to actively transport foreign particles to the perimeter of the eye's exposed surface. The particles are then collected in the lacrimal lake at the inside edge of the eye where they are flushed from the eye with tears. The eye maintains adequate vision throughout this process due to the brevity of the blinking action.

\subsection{Exploring Eye-Inspired Solutions}

Directly implementing many parts of the human eye analogy is ineffective for this problem. Use of fluids in the lunar environment is inappropriate due to the hard vacuum and large temperature ranges present. The use of a mechanical wiper is unsuitable due to the abrasiveness of the particles and the sensitivity of the protected optical surfaces.

Transferable strategies include transporting particles to the perimeter of sensitive surfaces, and actively protecting said surfaces while in operation.

These strategies, combined with the strategy of using charge to repel and attract, identified by the keyword 'repel', inspired the use of high-voltage electric fields to transport and contain particles around the perimeter of the LIDAR receiver. This method allows for dust protection while in operation (cover opened), and also exploits the fact that lofted lunar 
dust always carries an electrostatic charge (in the absence of mechanical disturbance).

\section{Prototype Design}

A prototype dust protection system was developed based on the analogies and concepts described previously. The prototype is comprised of two main assemblies: a passive subsystem consisting of an SMA actuated, bivalve-inspired lid assembly, and an active protection subsystem consisting of an integral DC field generator in a circular collar, inspired by the charge-based strategy. Figure 3 shows the prototype assembly in its closed position.

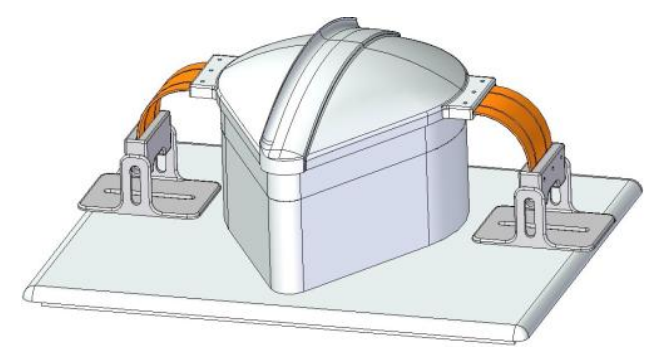

Figure 3. Prototype assembly in the closed position.

\subsection{Passive Subsystem}

The bivalve-inspired lid was developed with a focus on meeting the challenges introduced by the associated configuration, and in particular, the central seam between the two halves. An overlap design protects the central seam where the two lid sections meet, preventing any particles from falling onto the LIDAR equipment upon opening. A pointed lip is fashioned at the outside edge of the overlap section to guide dust away from the edge of the lid while it is in the closed position. The underside edges of both lid sections have a mating groove that fits the top of the base part, ensuring alignment when in the closed position. The base is a stationary part that encases the LIDAR equipment and is shaped to fit the two-part lid in its closed position.

Lid-section actuation is accomplished by a pair of novel SMA actuators, one for operation of each lid section. Heat is supplied to each actuator by three independently controlled thin-film surface heaters. The SMA used for this prototype is a Ni-Ti alloy known as Nitinol. The goal is to provide robust and repeatable actuation of both lids. These actuators are designed to lift the lid sections from a horizontal, seated position (approx. $90^{\circ}$ to the vertical) to a fully open position out of the LIDAR field of view (approx. $0^{0}$ to the vertical). Past designs have accomplished this motion through the use of a small step motor, causing the lid section(s) to rotate about a mechanical pivot. However, the many components of the step motor and pivot tend to be susceptible to dust intrusion, leading to decreased operability and lifetime.

To produce a cyclical opening and closing motion, an arrangement of three Nitinol strips is used, with the center strip being twice as wide as the outer strips. The strips are first annealed at $550^{\circ} \mathrm{C}$ for ten minutes, which determines the shape they will actively return to when heated. The outer strips are annealed in the flat position and the inner strip is annealed with a $110^{\circ}$ large radius bend, centered along the length of the strip. The strips are allowed to cool and can then be deformed plastically into matching configurations, i.e., flat or matching the inner strip. This antagonistic arrangement allows two-way motion to be achieved. If the hinge begins in the open position, with all strips being in-plane as shown in Figure 4, the central strip can be heated above a certain actuation temperature and will deform and return to its annealed shape. As it deforms, the central strip will force the outer strips to deform in the same manner. The central strip is then allowed to cool and will maintain its position, with minimal elastic recoil. At this point the outer strips can be heated causing them to deform and return to their annealed shape, i.e., flat, while also forcing the central strip back to this position. A return to the open position demonstrates two-way cyclical motion.

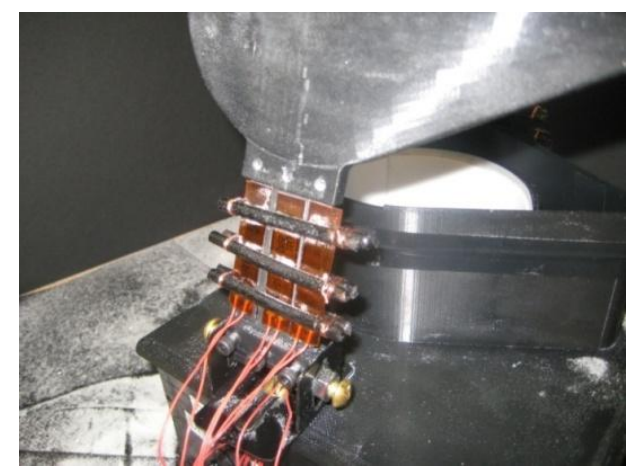

Figure 4. A complete actuator in the open position.

\subsection{Active Subsystem}

The receiver section of the base contains two semicylindrical insulated aluminum sheets. These sheets are oppositely charged to a $20 \mathrm{kV}$ relative electric potential, allowing them to store energy in an electric field. The system functions to deflect charged particles away from the lens face by exerting a force on them proportional to their charge.

As a charged particle enters the area influenced by the electric field, some stored energy in the field is expended as work exerted on the particle. The particle 
is drawn towards the oppositely charged plate along the field lines until it contacts the plate and is held there by electrostatic attraction and triboelectric forces. The active system is insulated from the receiver base with polytetrafluoroethylene (PTFE). Figure 5 shows the potential within the electric field.

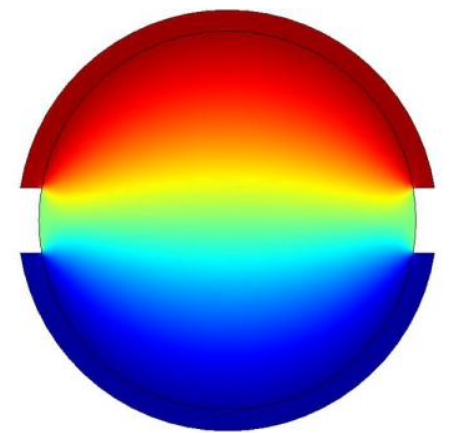

Figure 5. Active subsystem potential. $($ Red $=10 \mathrm{kV}$ Blue $=-10 \mathrm{kV})$

\section{Preliminary Testing}

Both prototype subsystems were subjected to preliminary testing. This testing served to provide a clearer picture of the capabilities and limitations of the technologies developed during the design phase of the project.

\subsection{Passive Subsystem Testing}

The passive subsystem, consisting of the SMA actuated lids coupled with the instrument base, prevents dust from entering the lens enclosure when the instrument is not operating. For this to happen, the lid sections must mate and seal effectively while shedding dust away from the lens upon opening. Also, the SMA actuation system must effectively open and close the lids through multiple cycles.

\subsubsection{Particle testing}

Particle intrusion tests were performed on the bivalveinspired lid as a means of evaluating the mating surface effectiveness and the ability of the lids to shed dust upon opening. Table 1 shows the results of testing with two different types of regolith simulant. Lid profiles were manufactured using a deposition-type rapid prototyping machine. The lid-and-base assembly was placed in the closed configuration (as shown in Figure 3) and subjected to repeated intrusion attempts with two types of lunar regolith stimulants (sand and flour). Lid performance was evaluated based on the percentage obscurement of the inner lens area by intruding particles upon opening.
Table 1. Results of particle intrusion testing.

\begin{tabular}{|c|c|c|}
\hline Testing Media & $\begin{array}{c}\text { Intrusive Particle } \\
\text { Count Per Cycle } \\
(\text { mean } \mathbf{n}=\mathbf{1 0})\end{array}$ & $\begin{array}{c}\text { \% Lens } \\
\text { Obscurement } \\
\text { Per Cycle }\end{array}$ \\
\hline Sand $\left(\mathbf{D}_{\text {mean }}=\sim \mathbf{0 . 1 m m}\right)$ & 16.4 & 0.0053 \\
\hline Flour $\left(\mathbf{D}_{\text {mean }}=\mathbf{5 0} \boldsymbol{\mu m}\right)$ & 283 & 0.023 \\
\hline
\end{tabular}

Small quantities of each simulant were observed to settle on the lens area after repeated intrusion attempts. These representative particles would carry a static charge in the lunar environment and could be deflected away from the lens by the active subsystem.

\subsubsection{Actuator testing}

Under preliminary testing, the SMA actuation system was capable of greater than one-hundred repeatable lid-opening and closing cycles. Thin film heaters were used to heat the SMA strips to their actuation temperature, using (on average) 8 Watts of power during actuation. The strips were allowed to cool for approx. two minutes between actuations. After an initial break-in period, where a small loss of range of motion was observed (approximately $30^{\circ}$ ), the actuator was cycled open and closed one hundred times. An $80^{\circ}$ range of motion was observed for the remainder of the test. With this range of motion, the SMA actuation system was still capable of opening the lids outside of the field of view of the LIDAR.

\subsection{Active Subsystem Testing}

Charged polystyrene particles $\left(D_{\text {mean }}=1 \mathrm{~mm}\right)$ were used to test the active subsystem. The particles were imparted with a weak static electrical charge, then were dropped from approximately $0.5 \mathrm{~m}$ above the active system. Their paths were observed to deflect in the electric field, with the particles adhering to one of the plates, depending on the polarity of their charge. A small percentage (1\% to $2 \%$ ) of the particles did not receive sufficient static charge and did not collect on the receiver collar. Peak power use for the active system was measured to be approx. $200 \mathrm{~mW}$.

\section{Discussion}

Preliminary testing results helped to reveal the strengths and limitations of the concepts drawn from the biomimetic design process.

Strengths of the overall design include: light weight and high resistance to dust of the SMA actuation system; effective passive protection offered by the bivalve-inspired lid design and supplementary 
protection offered by the active subsystem; low power requirements; and very few moving parts.

Limitations of the design include the relatively long cycle times and loss of stroke of the actuation system. The loss of stroke could be mitigated by proper adjustment and cycling of the actuators before deployment. In addition, the particular $\mathrm{Ni}-\mathrm{Ti}$ alloy used for testing has an actuation temperature range too low for use on the lunar surface. Refinement of this design could include selection of more exotic SMAs with higher actuation temperatures, or the introduction of a temperature regulation system for the actuators.

With respect to the use of biologically meaningful keywords and the resulting analogical transfer, this case study highlights the advantages of the use of analogies identified by a systematic search versus those that are more obviously relevant. The direct keyword 'protect' led to the bivalve-inspired lid configuration and SMA actuation of the lid. The keyword 'repel' falls under the category of enabling keywords, i.e., repel is an action that enables protection [2]. This led to the use of an electric field to repel regolith from surfaces to be protected. The obviously relevant biological analogy to protecting a visual sensing system is the human eye. However, direct implementations of applicable strategies, e.g., the use of fluid and wiping action, were unsuitable.

The complexity of the lunar environment and regolith are such that the design of solutions requires an iterative search and development of innovative ideas. An initial search identified potential solutions, but most of these solutions posed specific challenges. The problems that resulted from potential solutions then required further search and development to identify corresponding solutions.

For example, the two-piece lid solution stemming from the bivalve analogy is likely to reduce the amount of regolith that may fall onto the lens during opening and closing over the existing solution. However, new challenges that follow include protecting the seal between the two halves of the lid, and protecting an additional hinge that results.

This iterative process is essential to solve complex problems such as those posed by lunar regolith.

\section{Summary and Further Work}

A biomimetic search led to innovative solutions for the complex problem of protecting mechanisms from lunar dust. The solutions stemming from the biomimetic study were prototyped, and found to function effectively during preliminary testing.

Further work should include the refinement of the developed concepts into a fully Moon-compatible, detailed design. Further testing is also required in simulated lunar conditions, as well as with more advanced lunar regolith stimulants. Lastly, more detailed study of the long-term behaviour of the SMA actuation system in lunar conditions is required.

This study supports the usefulness of biological keyword searches using functional and related engineering keywords, as compared to application of obvious biological analogies. Therefore, continuing work on developing a systematic method of identifying and applying relevant biological analogies to design is underway.

\section{Acknowledgements}

We are grateful for the financial support of both the Natural Sciences and Engineering Research Council of Canada and the Department of Mechanical Engineering at Dalhousie University.

\section{References}

[1] Chiu, I., Shu, L.H., "Biomimetic Design Through Natural Language Analysis to Facilitate Cross-domain Information Retrieval", Artificial Intell. for Eng. Des. Analysis \& Manufacturing, 2007, 21/1:45-59.

[2] Cheong, H.M., Shu, L.H., Stone, R., McAdams, D., "Translating Terms of the Functional Basis into Biologically Meaningful Keywords", Proc. ASME Int. Design Engineering Technical Conferences, NYC, 2008, DETC2008-49363 (DTM).

[3] Stone, R.B., and Wood, K.L, "Development of a Functional Basis for Design," Journal of Mechanical Design, Transactions of the ASME, 2000, 122, pp. 359-369.

[4] Zakrajsek, J., McKissock, D., Woytach, J., Oswald, F., McEntire, K., Hill, G., Abel, P., Eichenberg, D., Goodnight, T., "Exploration Rover Concepts and Development Challenges", Proc. of 1st AIAA Space Exploration Conference, Orlando, FL, 2005, AIAA-2005-2525.

[5] Stubbs, T.J., Vondrak, R.R., Farrell, W.M., "Impact of Dust on Lunar Exploration", Proceedings of Dust in Planetary Systems, 2005.

[6] Purves, W., Sadava, D., Orians, G., Heller, H., Life, The Science of Biology, 6/e, Sinauer Associates, Sunderland, MA, 2001.

[7] Sturm, C. F., Pearce, T. A., and Valdez, A., eds., The Mollusks: A Guide to Their Study, Collection, and Preservation, American Malacological Society, 2006.

[8] Drake R.L., Vogl W., And Mitchell A. W. M., Gray's Anatomy for Students. Churchill Livingstone, 2005, pp. 831835. 